\title{
A characteristic-based split meshless local Petrov-Galerkin method for unsteady three-dimensional incompressible fluid flow
}

\author{
J. Mužík \& K. Kováŕík \\ Faculty of Civil Engineering, Department of Geotechnics, \\ University of Žilina, Slovakia
}

\begin{abstract}
A meshless local Petrov-Galerkin (MLPG) method has been developed for solving 3D incompressible isothermal laminar flow problems. It is derived from the local weak form of the Navier-Stokes equations by using the general MLPG concept. By incorporating the multi quadrics radial basis function (MQ-RBF) approximations for trial functions, the local weak form is discretized, and is integrated over the local subdomain for the unsteady incompressible fluid flow analysis. The present numerical technique uses characteristic-based split algorithm to solve Navier-Stokes equations in terms of primitive variables. A test case of lid-driven cavity flow is presented. The numerical procedure produces stable solutions with results comparable to those of other conventional methods.

Keywords: meshless analysis, meshless Petrov-Galerkin method, Navier-Stokes equation.
\end{abstract}

\section{Introduction}

The solving of incompressible Navier-Stokes equations together with the continuity equation constitutes a major problem of computational fluid dynamics. The mesh-based finite element (FEM) and volume (FVM) methods are usually used to solve this problem. However, these equations are nonlinear, which leads to the simulation usually requiring large meshes and lengthy computation [1].

The success of mesh-based methods relies on the mesh quality. Thus, their numerical simulations are often affected by mesh generation, especially for the 
problems with complex geometry. Recent development in the automatic mesh generation procedures for mesh-based methods relieves the difficulties. However, to maintain detailed data about the mesh, including all nodal and element relations is still very expensive. These make mesh generation, modification, and re-meshing a time consuming process.

To overcome the above mentioned difficulties, a number of meshless methods have been developed in the past two decades, in seeking to avoid the drawbacks or weakness of the standard numerical methods. From the viewpoint of approximation techniques, many meshless methods are based on the moving least square (MLS). Another popular meshless approximation technique is radial basis functions (RBF). Initially, RBFs were developed for multivariate data and function interpolation. It was found that RBFs were able to construct an interpolation scheme with favorable properties such as high efficiency, good quality and capability of dealing with scattered data, especially for higher dimension problems.

The meshless local Petrov-Galerkin method (MLPG) like other meshless methods, is based on regularly or randomly distributed nodal points covering the domain. Every node is at the centre of a surrounding local mesh of simple shape (quadrilateral, circle, sphere etc.). The unknown variable in this point is then expressed using a local weak formulation of governing equation on this local mesh. All of these unknown variables are approximated by RBF interpolation method (e.g. $[2,3])$ to obtain a system of linear equations. Solving this system of equations leads to a numerical solution of the problem.

The MLPG method was first applied to solve the incompressible flow equations by Lin and Atluri [4], who reported the use of the direct solution of a system of non-linear equations together with an upwind scheme to overcome oscillations produced by the convection term. They also added the perturbation term to the continuity equation to satisfy the Babuska-Brezzi condition. However, the problem remains of finding a proper value of the perturbation parameter in cases of flow with higher Reynolds numbers. Meshless methods can also be aided by the use of CBS to overcome the problem of the non-linearity of the Navier-Stokes equations in primitive variables (e.g. [5]). On the other hand approaches developed using the vorticity-stream or the velocity-vorticity formulation cannot be directly extended to solve in $3 \mathrm{D}$, and some types of boundary conditions are also difficult to define. Therefore, the primitive variables method is applied in this article.

It is interesting to explore whether the MLPG method has a potential to solve three-dimensional (3D) flow problems as efficient as two-dimensional case. This motivates the present paper. In the study, the time integration of the Navier-Stokes equations in the primitive variable form is carried out by means of the fractional step procedure, in which the momentum equations and the Poisson equation for pressure are solved separately at each time step. This procedure is applied to threedimensional incompressible lid-driven cavity flow with Reynolds numbers of 100 , 400 , and 1000 . The computed velocity profiles along the vertical and horizontal centrelines were given and compared with the results of Ding et al. [6]. 


\section{Governing equations and a characteristic-based split algorithm}

The governing equations for unsteady incompressible viscous fluid flow are Navier-Stokes equations in the primitive variable form can be written as

$$
\begin{gathered}
v \frac{\partial^{2} u_{i}}{\partial x_{j} \partial x_{j}}-\frac{\partial\left(u_{j} u_{i}\right)}{\partial x_{j}}-\frac{1}{\rho} \frac{\partial p}{\partial x_{i}}-\frac{\partial u_{i}}{\partial t}=f_{i} \\
\frac{\partial u_{i}}{\partial x_{i}}=0
\end{gathered}
$$

where $u_{i}$ is the velocity in $i$ direction, $p$ is the pressure, $f_{i}$ is the body force in direction $i, v$ is the kinematic viscosity and $\rho$ represents the density of fluid. Equation (1) is the momentum equation and equation (2) is the continuity equation. A CBS algorithm is used to solve this problem (see [5, 7]). The time derivative of the velocity vector in a momentum eqn. 1 can be replaced with a difference approximation and following relation is obtained

$$
u_{i}^{n+1}=u_{i}^{n}+\Delta t\left[v \frac{\partial^{2} u_{i}}{\partial x_{j} \partial x_{j}}-\frac{\partial\left(u_{j} u_{i}\right)}{\partial x_{j}}-f_{i}+\frac{\Delta t}{2} u_{k} \frac{\partial}{\partial x_{k}}\left(\frac{\partial\left(u_{j} u_{i}\right)}{\partial x_{j}}+f_{i}\right)\right]^{n}-\frac{\Delta t}{\rho} \frac{\partial p^{n+1}}{\partial x_{i}}
$$

where upper indexes $n$ and $n+1$ indicate the time step, $v$ is the kinematic viscosity and $\Delta t$ is the length of the time interval. The last term in square brackets acts as the stabilizing term (see [5]). Equation (2) is an explicit formula for the convection and viscous terms, and an implicit one for the pressure term. Equation (2) is simplified using the fractional time step approximation (e.g. [6, 8]), which computes the intermediate velocity $\tilde{\mathbf{u}}$ using simplified momentum equation

$$
\tilde{u}_{i}=u_{i}^{n}+\Delta t\left[v \frac{\partial^{2} u_{i}}{\partial x_{j} \partial x_{j}}-\frac{\partial\left(u_{j} u_{i}\right)}{\partial x_{j}}-f_{i}+\frac{\Delta t}{2} u_{k} \frac{\partial}{\partial x_{k}}\left(\frac{\partial\left(u_{j} u_{i}\right)}{\partial x_{j}}+f_{i}\right)\right]^{n}
$$

When we compare (3) and (4) we get

$$
u_{i}^{n+1}=\tilde{u}_{i}-\frac{\Delta t}{\rho} \frac{\partial p^{n+1}}{\partial x_{i}}
$$

The intermediate velocity $\tilde{u}_{i}$ does not satisfy the continuity equation (2). The velocity components $u_{i}^{n+1}$ must satisfy the continuity equation which implies

$$
\frac{\partial^{2} p^{n+1}}{\partial x_{i} \partial x_{i}}=\frac{\rho}{\Delta t} \frac{\partial \tilde{u}_{i}}{\partial x_{i}}
$$

Equation (6) is the Poisson's equation with non-zero source term [1]. The equation (4) and equation (5) are solved explicitly by updating nodal values for velocity components. The pressure equation (6) is solved using MLPG over problem domain with boundary conditions $p^{n} \mid \Gamma_{u}=\bar{p}^{n}$ and $\partial p^{n} / \partial n \mid \Gamma_{q}=\bar{q}^{n}$. 


\section{The MLPG method formulation}

The meshless local Petrov-Galerkin method (MLPG) is truly meshless method which requires no elements or global background mesh, for either interpolation or integration purposes. The area of interest $\Omega$ with the boundary $\Gamma$ is covered by points within the area and also on the global boundary (see Figure 1). Consider a local quadrilateral sub-domain $\Omega_{s}$ with boundary $\Gamma_{s}$ centred at every point $s$. This sub-domain is regular around all the internal points, but at the points on the global boundary this local boundary consists of a part of the global boundary intersected with the local sub-domain $\Gamma_{s}$. To express the local boundary integral form of the Navier-Stokes equations using the CBS algorithm in a domain $\Omega_{s}$, we apply the weighting residual principle to equation $4-6$. For simplicity we neglect a body force term to obtain the following weak form:

$$
\begin{gathered}
\int_{\Omega_{s}} w \tilde{u}_{i} d \Omega_{s}=\int_{\Omega_{s}} w u_{i}^{n} d \Omega_{s}+\Delta t \int_{\Omega_{s}} w\left[v \frac{\partial^{2} u_{i}}{\partial x_{j} \partial x_{j}}-\frac{\partial\left(u_{j} u_{i}\right)}{\partial x_{j}}+\frac{\Delta t}{2} u_{k} \frac{\partial}{\partial x_{k}}\left(\frac{\partial\left(u_{j} u_{i}\right)}{\partial x_{j}}\right)\right]^{n} d \Omega_{s} \\
\int_{\Omega_{s}} w u_{i}^{n+1} d \Omega_{s}=\int_{\Omega_{s}} w \tilde{u}_{i} d \Omega_{s}-\frac{\Delta t}{\rho} \int_{\Omega_{s}} w \frac{\partial p^{n+1}}{\partial x_{i}} d \Omega_{s} \\
\int_{\Omega_{s}} w \frac{\partial^{2} p^{n+1}}{\partial x_{i} \partial x_{i}} d \Omega_{s}=\frac{\rho}{\Delta t} \int_{\Omega_{s}} w \frac{\partial \tilde{u}_{i}}{\partial x_{i}} d \Omega_{s} \\
w(q)=\left\{\begin{array}{cc}
1-6 q^{2}+8 q^{3}-3 q^{4} & q \leq 1 \\
0 & q>1
\end{array}\right.
\end{gathered}
$$

a)

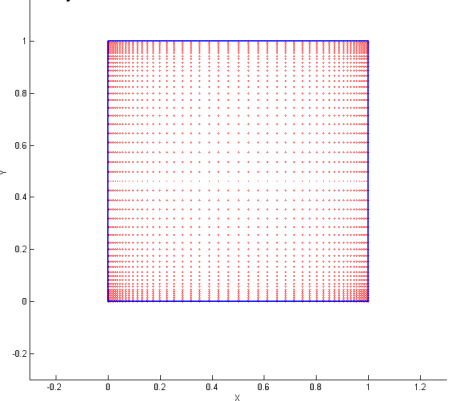

b)

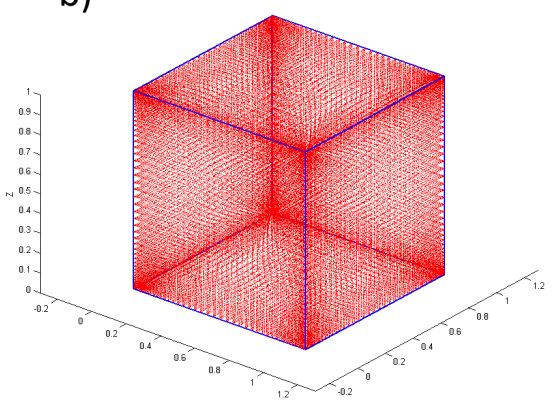

Figure 1: Schema and nodal distribution for $3 \mathrm{~d}$ lid-driven cavity flow, a) top view and b) axonometric view of the model.

Here $w$ is a weighting (test) function. The test function is chosen to be the 4th order spline function defined by (10) where $q$ is Euclidian distance from actual node normalized by local quadrature (mesh) dimensions. After integration by parts in (7) and (9) the following integral equations can be obtained (see also [8, 10, 18]): 


$$
\begin{aligned}
& \int_{\Omega_{s}} w \tilde{u}_{i} d \Omega_{s}=\int_{\Omega_{s}} w u_{i}^{n} d \Omega_{s}+\Delta t {\left[\begin{array}{l}
\int_{\Gamma_{s}} w \frac{\partial u_{i}}{\partial x_{j}} n d \Gamma_{s}-\int_{\Omega_{s}} \frac{\partial w}{\partial x_{j}} \frac{\partial u_{i}}{\partial x_{j}} d \Omega_{s}-\int_{\Omega_{s}} w \frac{\partial\left(u_{j} u_{i}\right)}{\partial x_{j}} d \Omega_{s}+ \\
\frac{\Delta t}{2} \int_{\Gamma_{s}} w u_{k} \frac{\partial\left(u_{j} u_{i}\right)}{\partial x_{j}} n d \Gamma_{s}-\frac{\Delta t}{2} \int_{\Omega_{s}} \frac{\partial w}{\partial x_{k}} u_{k} \frac{\partial\left(u_{j} u_{i}\right)}{\partial x_{j}} d \Omega_{s}- \\
\frac{\Delta t}{2} \int_{\Omega_{s}} w \frac{\partial u_{k}}{\partial x_{k}} \frac{\partial\left(u_{j} u_{i}\right)}{\partial x_{j}} d \Omega_{s}
\end{array}\right] } \\
& \int_{\Gamma_{s}} w \frac{\partial p^{n+1}}{\partial x_{i}} n d \Gamma_{s}-\int_{\Omega_{s}} \frac{\partial w}{\partial x_{i}} \frac{\partial p^{n+1}}{\partial x_{i}} d \Omega_{s}=\frac{\rho}{\Delta t} \int_{\Omega_{s}} w \frac{\partial \tilde{u}_{i}}{\partial x_{i}} d \Omega_{s}
\end{aligned}
$$

The boundary integrals over $\Gamma_{s}$ in (11) and (12) needs to be evaluated only for nodes where local quadrature intersects with global boundary. Unknown values of the pressure, velocities and its derivatives in the arbitrary point can now be approximated by values of the pressure in $N$ neighbouring points using RBF interpolation. Multiquadrics RBFs are used here

$$
R\left(r_{i j}\right)=\left(r_{i j}^{2}+\left(\alpha_{c} d_{c}\right)^{2}\right)^{q}
$$

Here $R\left(r_{i j}\right)$ is the multiquadrics RBF between points $i$ and $j$ (see [1-3]), $r_{i j}$ is the Euclidian distance between these two points and $\alpha_{c}$ is the so-called shape factor of the multiquadrics function and $d_{c}$ is average nodal spacing around node of interest. A technique described in detail in [7] can be used to obtain a set of RBF shape functions $\Phi_{i j}$, which can be used to express the pressure, velocities and its derivatives in the point $i$ as

$$
p_{i}=\sum_{j=1}^{N} \Phi_{i j} p_{j}, \frac{\partial p_{i}}{\partial x_{k}}=\sum_{j=1}^{N} \frac{\partial \Phi_{i j}}{\partial x_{k}} p_{j}, u_{i}=\sum_{j=1}^{N} \Phi_{i j} u_{j}, \frac{\partial u_{i}}{\partial x_{k}}=\sum_{j=1}^{N} \frac{\partial \Phi_{i j}}{\partial x_{k}} u_{j}
$$

using these shape functions leads equations (11), (12) and (8), respectively, to become

$$
\begin{gathered}
\sum_{m=1}^{N} M_{s m} \tilde{u}_{i m}=\sum_{m=1}^{N} M_{s m} u_{i m}^{n}+\Delta t \sum_{m=1}^{N}\left[A_{s m} u_{i m}^{n}-B_{s m} u_{i m}^{n}-C_{s m} u_{i m}^{n}+D_{s m} u_{i m}^{n}-E_{s m} u_{i m}^{n}-F_{s m} u_{i m}^{n}\right] \\
\sum_{m=1}^{N} A_{s m} p_{m}^{n+1}-\sum_{m=1}^{N} B_{s m} p_{m}^{n+1}=\frac{\rho}{\Delta t} \sum_{m=1}^{N} G_{s m} \tilde{u}_{i m} \\
\sum_{m=1}^{N} M_{s m} u_{i m}^{n+1}=\sum_{m=1}^{N} M_{s m} \tilde{u}_{i m}-\frac{\Delta t}{\rho} \sum_{m=1}^{N} G_{s m} \tilde{u}_{i m}
\end{gathered}
$$

where $u_{i m}, \tilde{u}_{i m}$ and $p_{m}$ are the values of velocity components, intermediate velocity components and pressure, respectively, in the points neighbouring the reference point $s$. The matrices $M_{s m}, A_{s m}, B_{s m}, C_{s m}, D_{s m}, F_{s m}$ and $G_{s m}$ can be written as

$$
\begin{aligned}
& M_{s m}=\sum_{n=1}^{n_{e}} \int_{\Omega_{s n}} w_{s} \Phi_{m} d \Omega_{s n}, A_{s m}=\sum_{n=1}^{n_{e}} \int_{\Gamma_{s n}} w_{s} \frac{\partial \Phi_{m}}{\partial x_{j}} n d \Gamma_{s n}, B_{s m}=\sum_{n=1}^{n_{e}} \int_{\Omega_{s m}} \frac{\partial w_{s}}{\partial x_{j}} \frac{\partial \Phi_{m}}{\partial x_{j}} d \Omega_{s n}, \\
& C_{s m}=\sum_{n=1}^{n_{e}} \int_{\Omega_{s m}} w_{s} u_{j} \frac{\partial \Phi_{m}}{\partial x_{j}} d \Omega_{s n}, D_{s m}=\frac{\Delta t}{2} \sum_{n=1}^{n_{e}} \int_{\Gamma_{s n}} w_{s} u_{k} u_{j} \frac{\partial \Phi_{m}}{\partial x_{j}} n d \Gamma_{s n}, \\
& E_{s m}=\frac{\Delta t}{2} \sum_{n=1}^{n_{e}} \int_{\Omega_{s m}} \frac{\partial w_{s}}{\partial x_{k}} u_{k} u_{j} \frac{\partial \Phi_{m}}{\partial x_{j}} d \Omega_{s n}, \\
& F_{s m}=\frac{\Delta t}{2} \sum_{n=1}^{n_{e}} \int_{\Omega_{s m}} w_{s} \frac{\partial u_{k}}{\partial x_{k}} u_{j} \frac{\partial \Phi_{m}}{\partial x_{j}} d \Omega_{s n}, G_{s m}=\sum_{n=1}^{n_{e}} \int_{\Omega_{s m}} w_{s} \frac{\partial \Phi_{m}}{\partial x_{i}} d \Omega_{s n},
\end{aligned}
$$


where $n_{e}$ is the number of local quadrature subdomains. The mass matrix $M_{s m}$ can be used in either a full or lumped form. The lumped form is used here, because it eliminates a matrix inversion procedure. The upwind term matrices $D_{s m}, E_{s m}$ and $F_{\text {sm }}$ comes from last three terms in (11).

The fractional step algorithm described above can be summarized as follows

- Start with initial velocity field,

- Compute intermediate velocity field using (15) for each node,

- Solve the Poisson equation (16) using MLPG to obtain the pressure,

- Update velocities using (17).

\section{Numerical example}

In this section the MLPG solution of Navier-Stokes equations developed in the previous sections is validated by solving the $3 \mathrm{D}$ lid-driven cavity flow example as a benchmark problem of fluid flow simulation [6]. Lid-driven cavity flow is used as a standard test problem for the validation of numerical solutions of incompressible Navier-Stokes flow. The top wall of the cavity moves with a velocity $u_{x}=1$, and no-slip impermeable boundary conditions are assumed along the other three walls. The difficulty of this problem lies in the presence of singularities of pressure and velocity at the two upper corners of the cavity. Therefore, the density of points used increases toward the corners of the cavity (see Figure 1). The grid-point distribution in three directions is taken the same, and chosen as [8]

$$
x_{i}=\frac{1}{2}\left(1+\frac{\tanh \left[1.8\left(2 x_{i}^{u}-1\right)\right]}{\tanh [1.8]}\right)
$$

where subscript $i=1,2$ and 3 is the dimension and $x^{u}$ are the coordinates of the corresponding uniform distribution of points.

Based on CBS algorithm discussed in the previous section, the numerical simulation of the 3D lid-driven flow in a cubic cavity with Reynolds numbers of $R e=100, R e=400$ and $R e=1000$ are carried out. The initial values for all the variables at the interior points are set to zero. For the convergence criterion of steady flow, L2 norm of velocity difference between the new and old time levels is set to be less than $10 e-6$. Numerical simulations were performed on the grid of $41 \times 41 \times 41$ nodes. For all the numerical experiments carried out, the size of support domain is chosen as

$$
d_{s}=\alpha_{s} d_{c}
$$

where $d_{c}$ is average nodal spacing around point of interest and the shape parameter in the MQ RBFs is set to $\alpha_{c}=2.1$ and $q=1.03$. In order to examine the performance of MLPG method, the following profiles were computed: $u$-velocity along the vertical centerline and $v$-velocity components along the horizontal centreline in the plane of $z=0.5$. Since there is no available analytical expression for the solution, the numerical solutions of previous literature are adopted as references 
to validate the present results. The lid-driven flow in a cubic cavity has been studied by meshless local multiquadric-based differential quadrature (LMQDQ) method and reported by Ding et al. [6]. The computed results of the present method are compared with those of Ding et al. [6]. The velocity profiles of $u$ component along the vertical centerline and $v$ component along the horizontal centerline of the plane $\mathrm{z}=0.5$ are plotted in Figure $2 \mathrm{a})-\mathrm{c}$ ) for $R e=100,400$ and 1000 , respectively. It can be seen that the velocity profiles agree very well with those of Ding et al. [6]. It indicates that the MLPG method can achieve solution of equivalent accuracy as the other meshless method with the similar mesh size.

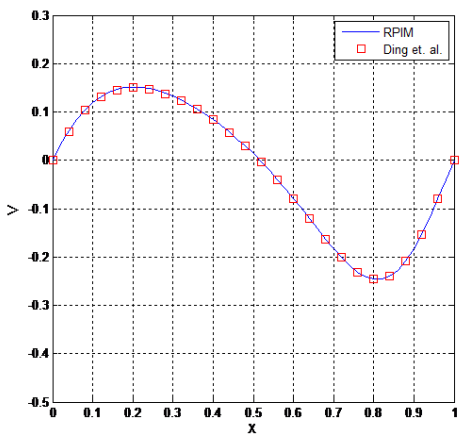

a)
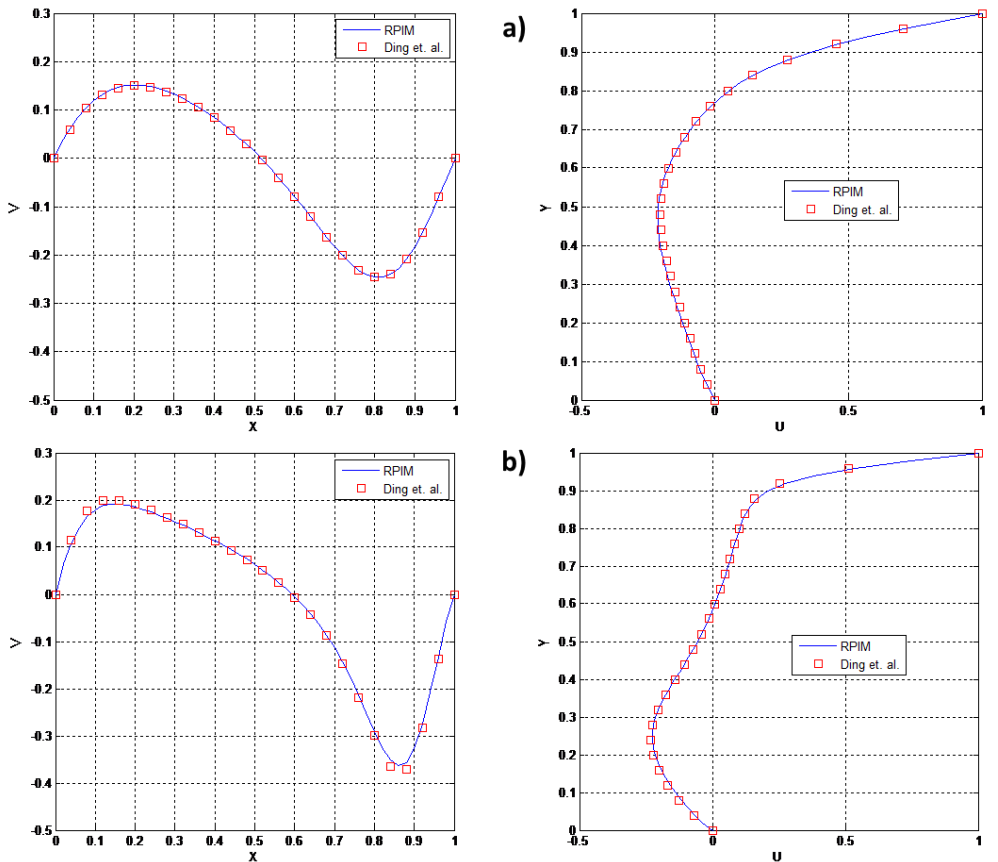

b)
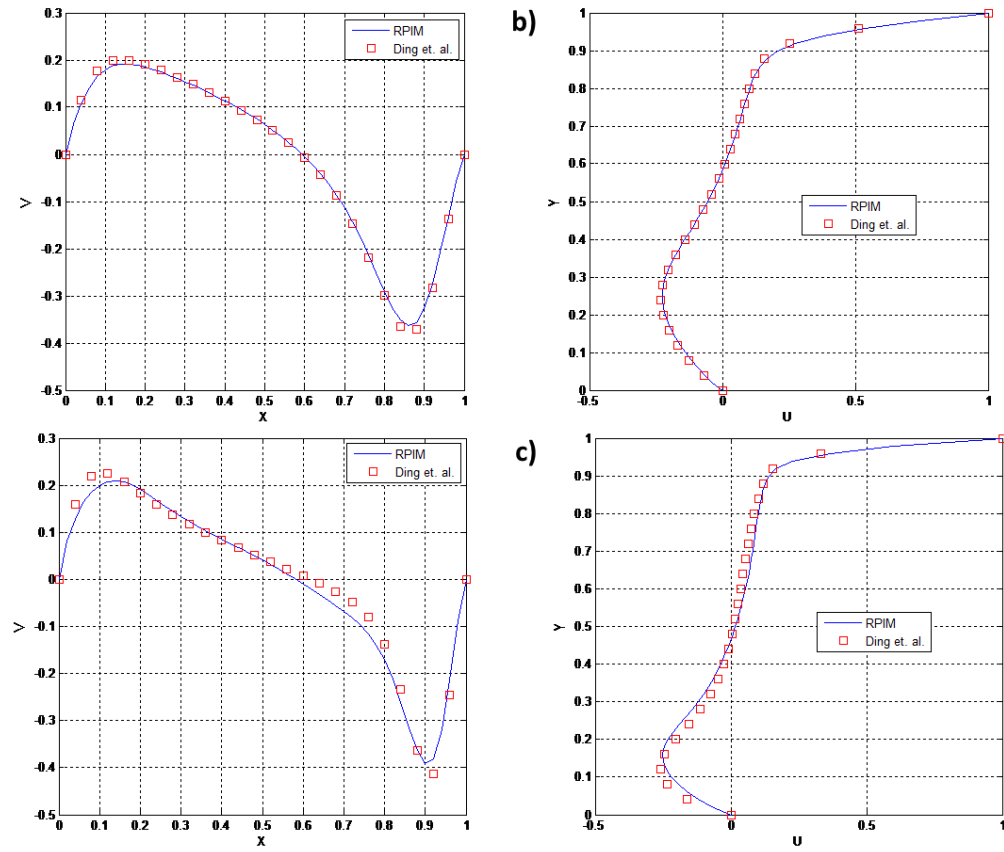

c)

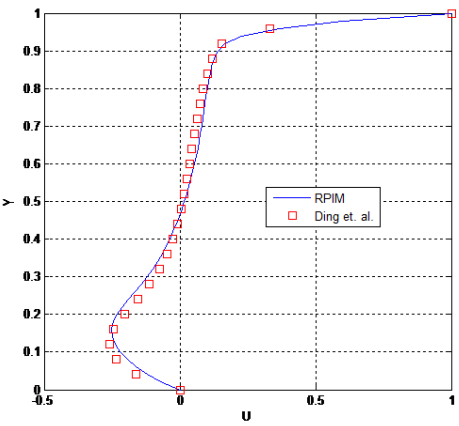

Figure 2: Comparison of velocity component distribution along the vertical centerline of cubic cavity ( $v-x$ and $u-y)$ : a) $R e=100$, b) $R e=400$, c) $R e=1000$. 
For the purpose of detailed flow visualization the flow field patterns are shown using three centroidal planes of the cube, which are located at $x=0.5, y=0.5$ and $z=0.5$. Two-dimensional planar projections of the velocity vector field at $R e=100$, 400 and 1000 on the three planes are shown in Figures 3-5. These figures illustrate the changing of flow patterns as Reynolds number varies from different viewing angles.
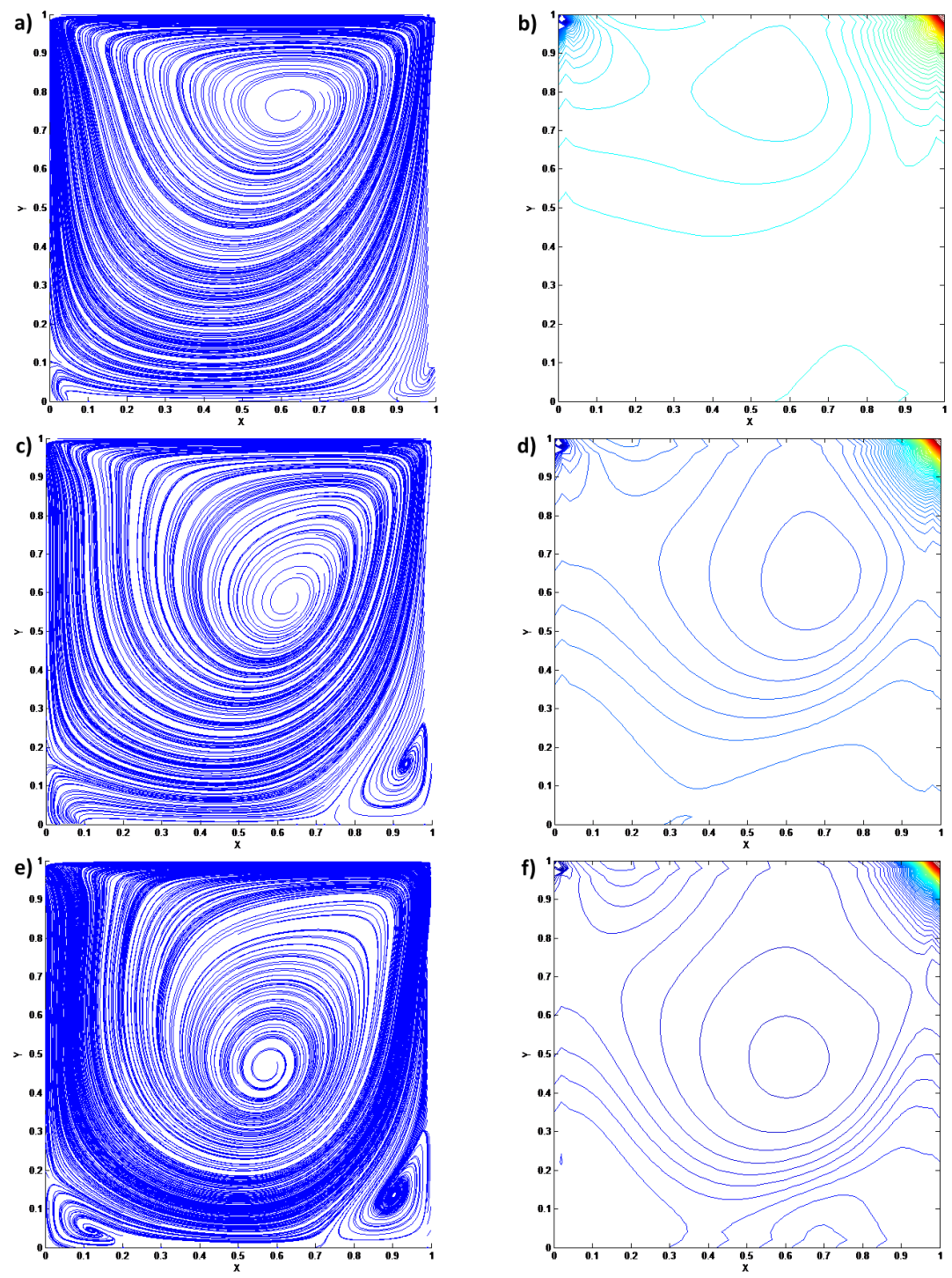

Figure 3: Flow pattern and pressure contours on mid-planes at $z=0.5$ : a) and b) $R e=100, \mathrm{c})$ and d) $R e=400, \mathrm{e}$ ) and f) $R e=1000$. 

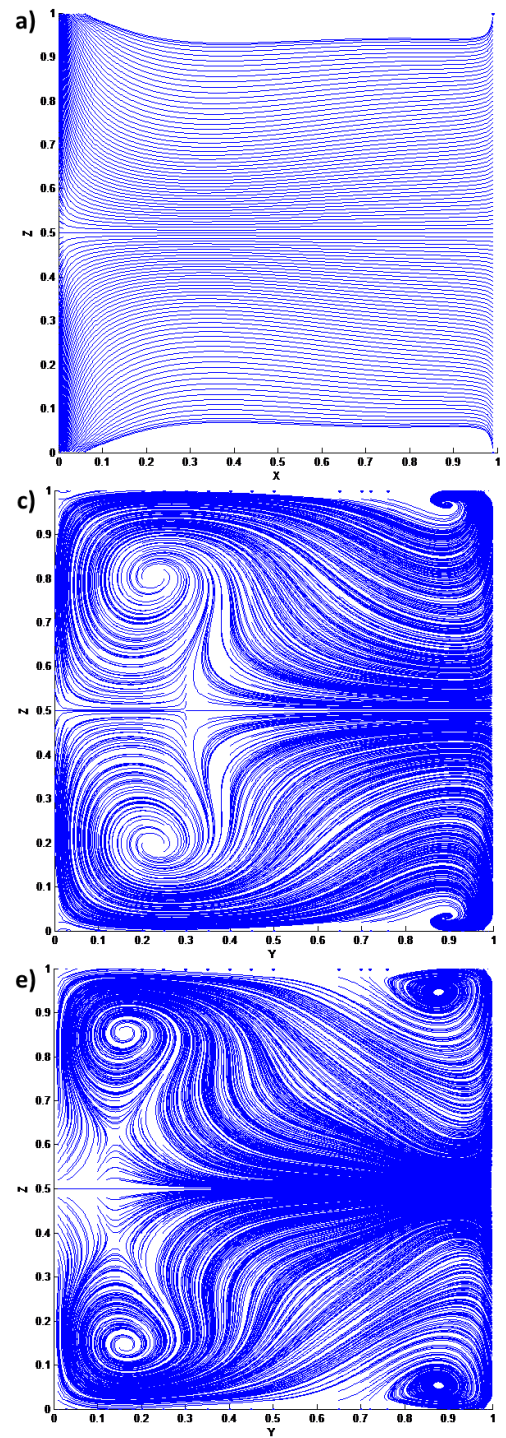
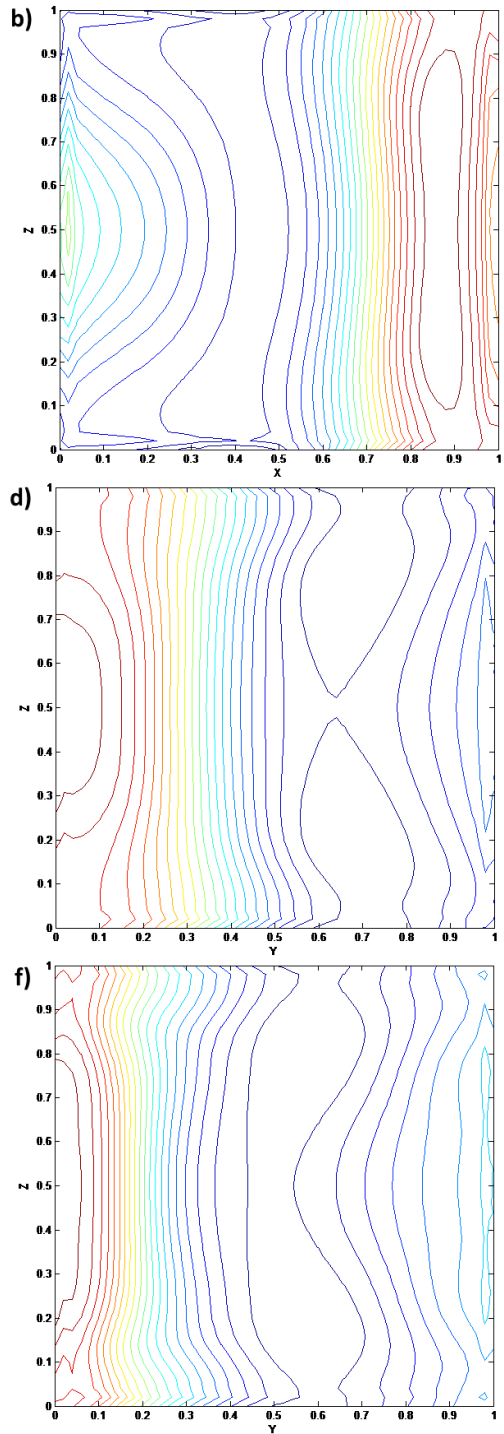

Figure 4: Flow pattern and pressure contours on mid-planes at $x=0.5$ : a) and b) $R e=100, \mathrm{c})$ and d) $R e=400, \mathrm{e})$ and f) $R e=1000$.

It can be observed from the flow pattern in the plane of $z=0.5$ (Figure 5) that the axis of the primary vortex starts in the upper right half region, then gradually moves towards the cube center as the Reynolds number increases. The flow also demonstrates strong three-dimensional characteristic. From the distribution of velocity vectors (Figures 3 and 4 ) in the planes of $x=0.5$ and $y=0.5$, it can be seen that secondary vortices move sidewards in the $y$-direction and upwards in the $z$ direction as the Reynolds number increases. 

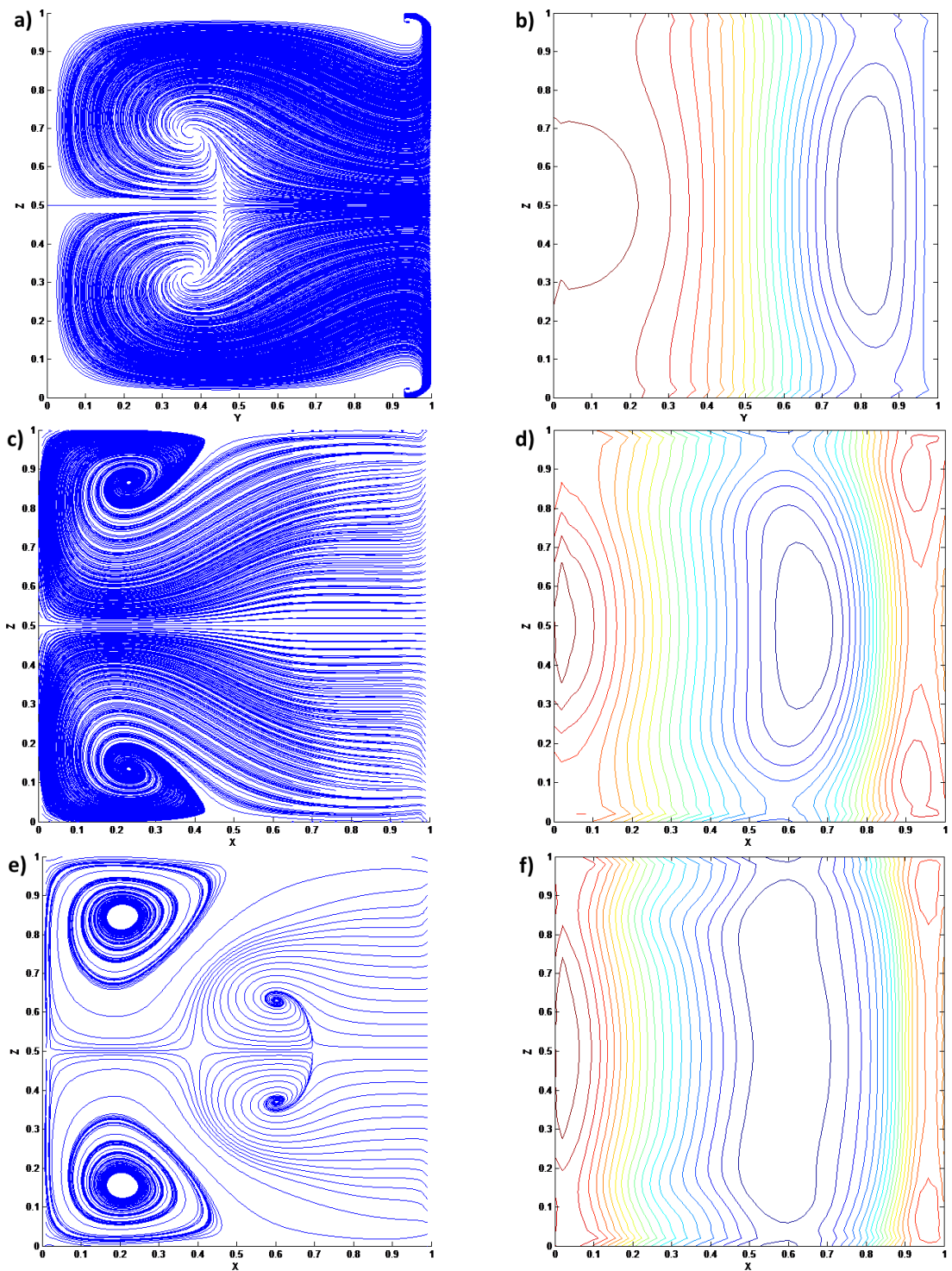

Figure 5: Flow pattern and pressure contours on mid-planes at $y=0.5$ : a) and b) $R e=100, \mathrm{c})$ and d) $R e=400, \mathrm{e})$ and f) $R e=1000$.

Similar flow patterns for $R e=100,400$ and 1000 were also observed and reported by Ding et al. [6], Fujima et al. [9] and Guj and Stella [10]. 


\section{Conclusions}

A possible use of the meshless MLPG with RBF interpolation is presented here for the computation of three-dimensional incompressible flows. The primitive variable formulation of the Navier-Stokes equations and the CBS scheme is used to achieve stable and accurate results. The suitability of this procedure is validated using Lid-driven cubic cavity flow problem, simulated at several Reynolds numbers. Numerical solutions are compared with previous ones in the literature, and found in good agreement. The results demonstrate that the method is effective and useful for the large-scale simulations of computational fluid dynamics (CFD), especially for three-dimensional cases. However, there are still some issues, which remain unsolved in the MLPG method. For example, the shape parameter of MQ RBF needs to be determined by end-user, and the choice of optimal value of shape parameter still relies on the tests or experiences.

\section{Acknowledgement}

This contribution is the result of the project supported by Scientific Grant Agency of Slovak Republic (VEGA) No. 1-1159-12.

\section{References}

[1] Kovarik, K., A meshless solution of two dimensional density-driven groundwater flow. Boundary elements and other mesh reduction methods XXXIII. Southampton, pp. 253-264, WIT Press, 2011.

[2] Liu, G.R., Meshfree methods: Moving beyond the finite element method. CRC Press, 2009, ISBN 1420082094.

[3] Shu, C., Ding, H., Yeo, K.S., Local radial basis function-based differential quadrature method and its application to solve two-dimensional incompressible Navier-Stokes equations. Comput. Methods Appl. Mech. Eng., 2003, 192, pp. 941-954.

[4] Lin, H., Atluri, S.N., The Meshless Local Petrov-Galerkin (MLPG) method for solving incompressible Navier-Stokes equations. Comput. Model. Eng. Sci., 2001, 2, pp. 117-142.

[5] Nithiarasu, P., Codina, R., Zienkiewicz, O.C., The characteristic-based split (CBS) scheme - unified approach to fluid dynamics. Int. J. Numer. Methods Eng., 2006, 66, pp. 1514-1546.

[6] Ding, H., Shu, C., Yeo, K.S., Xu, D., Numerical computation of threedimensional incompressible viscous flows in the primitive variable form by local multiquadric differential quadrature method. Comput. Methods Appl. Mech. Engrg., 2006, 195, pp. 516-533.

[7] Kovárík, K., Mužík, J., Sitányiová, D., A fractional step local boundary integral element method for unsteady two-dimensional incompressible flow. Eng. Anal. Bound. Elem., 2014, 44, pp. 55-63. 
72 Boundary Elements and Other Mesh Reduction Methods XXXVIII

[8] Najafi, M., Arefmanesh, A., Enjilela, V., Meshless local Petrov-Galerkin method-higher Reynolds numbers fluid flow applications. Eng. Anal. Bound. Elem., 2012, 36, pp. 1671-1685.

[9] Fujima, S., Tabata, M., Fukasawa, Y., Extension to three-dimensional problems of the upwind finite element scheme based on the choice of upand down-wind points, Comput. Methods Appl. Mech. Engrg., 1994, 112, pp. 109-131.

[10] Guj, G., Stella, F., A vorticity-velocity method for the numerical solution of 3D incompressible flows. J. Comput. Phys., 1993, 106, pp. 286-298. 